\title{
Natrum mur 200c promotes seed germination and increases total protein, chlorophyll, rubisco and sugar in early seedlings of cowpea under salt stress
}

\author{
Sandhimita Mondal, Nirmal Chandra Sukul and Soma Sukul (nee Chunari) \\ Department of Botany, Visva-Bharati University, Santiniketan, West Bengal, India.
}

\begin{abstract}
Background: High level of salinity deteriorates seed germination, growth and yield of crops in cultivated lands all over the world. There is no effective remedy to mitigate this global problem. Homeopathy offers a remedy like Natrum mur which at ultra high dilution ameliorates diseases of patients having strong desire for salt consumption. The purpose of the present study is to see whether potentized Natrum mur could alleviate salt stress in germinating seeds of cowpea Vigna unguiculata (L) Walp.

Methods: Water-soaked seeds were kept over moist filter paper in covered petridishes which were divided into 5 groups: (1) unstressed and untreated control in sterile distilled water, (2) in $100 \mathrm{mM}$ sodium chloride solution, (3) seeds pretreated with Natrum mur 200c and then kept in sterile distilled water, (4) seeds pretreated with Natrum mur 200c and then transferred to $100 \mathrm{mM} \mathrm{NaCl}$ solution and (5) seeds pretreated with $90 \%$ ethanol. Both Natrum mur $200 \mathrm{c}$ and its diluent medium $90 \%$ ethanol were diluted with distilled water 1:100 before use for treatment.
\end{abstract}

Results: Natrum mur 200c increased the rate of seed germination, seed water content and growth of seedlings. The drug also enhanced chlorophyll, soluble and insoluble sugar, rubisco and total protein content as compared to the untreated salt stressed group. Treatment with Natrum mur 200c increased salt tolerance in the seedlings as compared to the untreated salt stressed group. All the data were analyzed by ANOVA and the significance level was not less than $1 \%$.

Conclusion: Natrum mur 200c reversed the effects of salt stress in germinated seeds thereby providing evidence for Hahnemann's similia principle in plants. Potentized Natrum mur could be safely used with profit on plants grown on brackish soil.

Keywords: salinity; cowpea; germination; Natrum mur; growth, chlorophyll; rubisco.

\section{(c)) BY-NC-ND Licensed to GIRI}

Support: This study was supported by a grant from the Asiatic Society, Kolkata.

Conflict of interest: authors declare there is no conflict of interest

Received: 01 June 2012; Revised: 08 August 2012; Published: 30 September 2012.

Correspondence author: Nirmal Chandra Sukul, ncsukul@gmail.com

How to cite this article: Mondal S, Sukul NC, Sukul S. Natrum mur 200c promotes seed germination and increases total protein, chlorophyll, rubisco and sugar in early seedlings of cowpea under salt stress.. Int J High Dilution Res [online]. 2012 [cited YYYY Month dd]; 11(40):128-128. Proceedings of the XXVI GIRI Symposium; 2012 Sep 20-22; Florence (Italy). GIRI; 2012; Available from: http://www.feg.unesp.br/ ojs/index.php/ijhdr/article/view/570/578 